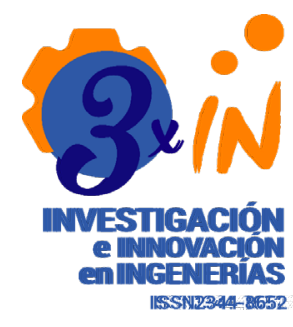

OPEN ACCESS

Recibido: 9/09/2020

Aceptado: 27/11/2020

Publicado: 04/01/2021

Correspondencia de autores: tfontalvoh@unicartagena.edu.co

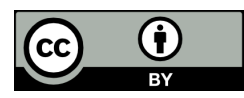

Copyrith 2020 by Investigación e Innovación en Ingenierias

\section{Enfoque sistémico convergente de la calidad aplicado a un programa académico de una Institución de Educación Superior}

\section{Convergent systemic approach to quality applied to an academic program of a Higher Education Institution}

\author{
Tomás José Fontalvo Herrera iD Yulibeth González Nieto iD \\ Universidad De Cartagena, Colombia
}

Resumen

Objetivo: PDiseñar a través de diferentes enfoques, herramientas y conceptos de calidad, una estructura de calidad que permita agregar valor y responder a las necesidades y expectativas específicas de los clientes implicados en el desarrollo del proceso de gestión de la movilidad académica saliente como parte del macroproceso de internacionalización del programa Administración Industrial de la Universidad de Cartagena, basado en el Enfoque Sistémico Convergente de la Calidad. Metodología: Para lo cual se realizó un estudio cualitativo y cuantitativo, que se soportó en la revisión documental y la observación del proceso. De igual manera se realizó un análisis cuantitativo y empírico para la aplicación de las herramientas de calidad en el objeto de estudio. Resultados: Se aporto una estructura de calidad sistémica que permitió integrar las diferentes herramientas de calidad para generar valor agregado en el proceso de internacionalización del programa. Conclusión: La aplicación del despliegue de la función de calidad (QFD), el análisis del modo y efecto de fallas (AMEF), la estandarización de procesos, los Poka Yoke y la matriz de valor agregado implementadas de forma sistémica, permite planificar y controlar el proceso de manera mas efectiva para su mejora y generación de valor.

Palabras clave: Calidad, procesos de internacionalización, herramientas de calidad, enfoque por procesos.

Abstract

Objective: Design, through different approaches, tools and concepts of quality, a quality structure that allows adding value and responding to the specific needs and expectations of the clients involved in the development of the outgoing academic mobility management process as part of the macroprocess of internationalization of the Industrial Administration program at the University of Cartagena, based on the Convergent Systemic Approach to Quality. Methodology. For which a qualitative and quantitative study was carried out, which was supported by the documentary review and the observation of the process. In the same way, a quantitative and empirical analysis was carried out for the application of quality tools in the object of study. Results. A systemic quality structure was provided that allowed the integration of the different quality tools to generate added value in the internationalization process of the program. Conclusion. The application of quality function deployment (QFD), failure mode and effect analysis (FMEA), process standardization, Poka Yoke and the value-added matrix implemented in a systemic way, allows planning and controlling the process in a more effective way for its improvement and generation of value.

Keywords: Quality, internationalization processes, quality tools, process approach.

Como citar (IEEE): T. Fontalvo-Herrera., Y. González-Nieto “Enfoque sistémico convergente de la calidad aplicado a un programa académico de una Institución de Educaciốn Superior", Investigación e Innovación en Ingenierías, vol. 9, n¹, 61-77, 2021. DOI: https://doi.org/10.17081/invinno.9.1.4069 


\section{Introducción}

En los últimos 25 años la dimensión internacional se ha posicionado como un elemento central de la educación en documentos internacionales, nacionales e institucionales; siendo además eje de discusión en las agendas gubernamentales, instituciones de educación superior (IES), organizaciones estudiantiles y agencias de acreditación al ser un nuevo indicador de calidad en la educación superior. El CNA [1] define el concepto de calidad aplicado al bien público de la educación superior como la síntesis de características que permiten reconocer un programa académico específico o una institución de determinado tipo y hacer un juicio sobre la distancia relativa entre el modo como en esa institución o en ese programa académico se presta dicho servicio y el óptimo que corresponde a su naturaleza.

En razón del cumplimiento de este último factor, la Universidad de Cartagena incluyó la internacionalización dentro de sus procesos estratégicos como factor clave para la satisfacción de los clientes, a través de la puesta en marcha de líneas de acción que incluyen las relaciones internacionales, la internacionalización del currículo, de la investigación, de la extensión y de la comunidad académica; viéndose reflejadas en las crecientes alianzas internacionales, movilidad académica de docentes, administrativos y estudiantes y vinculación en redes colaborativas internacionales de investigación [2].

El programa de Administración Industrial de la Universidad de Cartagena hace énfasis en la Internacionalización como un área estratégica, en la que se busca la consolidación de un programa académico de carácter global. Para alcanzar este objetivo, se definen como líneas de trabajo la movilidad internacional, la internacionalización del Plan de Estudios y el dominio de, por lo menos, una lengua extranjera [3]. Sin embargo, los esfuerzos para la internacionalización realizados por dicho programa académico deben adoptar una visión sistémica, en el que cada uno de sus procesos a partir de sus interrelaciones aporte en la consecución de los objetivos de visibilidad internacional.

Tal como lo define el BCSSS (Bertalanffy Center for the Study of Systems Science), un sistema es "algo mayor que la suma de sus partes porque consiste en esas partes, la manera en que se relacionan unas con otras y las cualidades emergentes de esas relaciones [4]. Para Jean Louis Le-Moigne un sistema activo es "un objeto dotado de fines u objetivos, que en un entorno bien delimitado, ejerce una actividad a la vez que ve evolucionar su estructura interna a lo largo del tiempo sin perder por ello su identidad" [5].

Lo anterior no lleva a generar las siguientes preguntas: ¿de qué modo es posible modelar los procesos y los servicios inmersos en el proceso de internacionalización del programa de Administración Industrial de la Universidad de Cartagena de tal modo que se genere valor agregado y se logre la satisfacción plena de sus clientes?, interrogante que constituye la razón de esta investigación, cuya respuesta se soporta en la aplicación de los distintos enfoques técnicos, herramientas de calidad y a los doce pasos para operacionalizar la intervención empresarial con el fin de poder analizar, representar, modelar, diseñar, implementar y sostener el Enfoque Sistémico Convergente de la Calidad (ESCC) planteado por Fontalvo [6]. 


\section{Marco teórico}

\section{Modelo conceptual de la calidad del servicio}

En [7] la calidad del servicio se define como una medida de qué tan bueno es el nivel del servicio entregado, coincidiendo con las expectativas del cliente. Almohaimmeed [8] afirma que la mayoría de las definiciones de servicios externos están de acuerdo con la definición de calidad de Parasuraman, en la cual la calidad del servicio se describió como la compatibilidad entre las percepciones y expectativas del cliente con el servicio real que experimentaron. Entregar un servicio de calidad significa estar conforme con las expectativas del cliente sobre una base consistente.

El conocimiento sobre la calidad de los bienes es insuficiente para entender la calidad del servicio, por tanto, existen algunos elementos determinantes de la calidad del servicio, como son:

- Fiabilidad: Implica consistencia de rendimiento y confiabilidad.

- Sensibilidad: Se refiere a la disposición de los empleados para brindar servicio.

- Competencia: Significa poseer las habilidades y conocimientos necesarios para realizar el servicio.

- Acceso: Implica accesibilidad y facilidad de contacto.

- Cortesía: Implica cortesía, respeto, consideración y amabilidad del personal de contacto.

- Comunicación: Significa mantener a los clientes informados en un lenguaje que puedan entender y escucharlos.

- Credibilidad: Implica confiabilidad, credibilidad, honestidad.

- Seguridad: Implica la seguridad física, la seguridad financiera, confidencialidad.

- Entender / conocer al cliente: Implica hacer el esfuerzo de comprender las necesidades del cliente.

- Tangibles: Incluye la evidencia física del servicio, como las facilidades físicas, apariencia de personal, herramientas o equipos utilizados para proporcionar el servicio, etc.

Sin embargo, varios elementos de la cultura interactúan con otras fuerzas externas y directa o indirectamente influyen en la satisfacción del cliente, lo que hace incluir este elemento en los aspectos a tener en cuenta.

\section{Enfoque por procesos}

La gestión orientada a los procesos de las empresas se basa en procesos de negocio. El enfoque basado en procesos permite no solo la descripción del negocio como una red de procesos interrelacionados, sino también el monitoreo continuo, la gestión y la mejora de los mismos. El alcance del modelado de procesos de negocio es amplio y aumenta continuamente. Los modelos de procesos comerciales se utilizan para la reingeniería y gestión de procesos, desarrollo de sistemas conscientes, soluciones de comercio electrónico, modelado de regulación empresarial, gestión del conocimiento, especificación de requisitos y otros fines [9]. La correcta identificación y la organización racional, la investigación oportuna y la evaluación de los procesos de negocio permiten identificar áreas problemáticas y facilitan la construcción de ventajas competitivas sostenibles [10]. 


\section{Enfoques técnicos y herramientas de calidad}

Uno de los modelos que genera la posibilidad de mirar la interdependencia de las variables a estudiar y la coexistencia de los enfoques de calidad utilizados para el mejoramiento o rediseño del proceso intervenido es el Enfoque Sistémico Convergente de la Calidad, definido por Fontalvo [6] en su artículo "Propuesta de intervención empresarial para la generación de valor en las organizaciones", lo define como "una metodología estructurada que facilita el diseño e implementación de modelos, programas y sistemas de gestión sobre los procesos, áreas u organizaciones, a través de la conjunción y articulación sistémica de los componentes del área de intervención como son: las estructuras de funcionamiento (ambiente activo), la interacción con el contexto, las finalidades del sistema, los enfoques técnicos y herramientas de calidad seleccionados, las especificaciones, las transformaciones a generar, la innovación y la convergencia del sistema", que se sustenta en doce pasos para su implementación, cuyas especificaciones serán abordadas más adelante.

Dentro de las herramientas de calidad más utilizadas que paoyan el ESCC se encuentra el Despliegue de la función calidad, o QFD por sus siglas en inglés Quality Function Deployment, creada por los profesores Akao y Mizuno en el año 1960, constituida por dos componentes. El primero se enfoca en el mejoramiento de la calidad de productos nuevos traduciendo las necesidades de los clientes en características de diseño y el desarrollo sistemático de las mismas en la manufactura y producción. El segundo componente está enfocado en el proceso de negocio de la organización, asegurándose que todas las operaciones, funciones y tareas hechas por las personas sean realizadas de la mejor manera de modo que se asegure la calidad [11]. Dichas funciones incluyen la planeación, el diseño, los prototipos, la producción u operaciones y el servicio [12]. Una de las principales herramientas de QFD para productos es la matriz, mejor conocida como "la casa de la calidad [13].

Por otro lado, existe una herramienta de mejora continua denominada Análisis del Modo y Efecto de la falla potencial (AMEF). El nombre de AMEF proviene del idioma inglés (Potential Failure Mode And Effect Analysis, FMEA). El AMEF es uno de los enfoques estructurados, sistemáticos y proactivos para el análisis de fallas de productos o sistemas. Un paso crítico del AMEF es identificar posibles modos de falla para subsistemas de productos, componentes y procesos, para los cuales el conocimiento del modo de falla de componentes es necesario como una fuente importante de conocimiento [14]. Es una técnica de ingeniería ampliamente utilizada para identificar y eliminar fallas conocidas y potenciales de sistemas, diseños, productos, procesos o servicios. Toda la información sobre factores de riesgo como ocurrencia (O), severidad (S) y detección (D) y sus pesos relativos se expresan en términos lingüísticos, representados por números [15]. En este sentido, cabe destacar que los mecanismos a prueba de fallas Poka Yoke son una herramienta similar, que hablan de errores y defectos, los cuales pueden prevenirse [16]. Bajo la premisa de Shingo [17], los errores son la causa de los defectos generados en el trabajo. Con el mecanismo Poka Yoke, una vez ocurrido el error y ser descubierto se puede llegar a resolver, evitando así que se convierta en un defecto. Los errores más comunes son procesos omitidos, fallas en el ajuste de las partes en un proceso, partes equivocadas o faltantes, instrucciones y herramientas mal preparadas, falta de instrucciones en el trabajo o complejidad como tal del mismo, errores humanos, que incluyen: olvido, falta de comprensión, falta de experiencia, descuido, lentitud entre otros. Por tal motivo un buen diseño del proceso impide en términos generales que ocurran los defectos, el cual pueden estar sustentado en inspecciones, automatización y sistemas Poka Yoke. 


\section{Metodología}

El presente artículo es una investigación de tipo descriptivo, cualitativo y cuantitativo, identificando las actividades que soportan el subproceso de Movilidad Internacional del programa Administración Industrial de la Universidad de Cartagena, contrastándose con las necesidades y expectativas de los cliente para proporcionar una modelación para su optimización bajo el Enfoque Sistémico Convergente de la Calidad planteado por Fontalvo [6] que se apoyo en la articulación de herramientas de calidad tales como: despliegue de la función calidad (QFD), análisis de modelo, efecto y falla (AMEF); mecanismos a prueba de fallas (poka-yoke) y matriz de valor agregado.

Para llevar a cabo la investigación, se realizó una revisión documental a Planes de Desarrollo del programa de Administración Industrial, informe de autoevaluación, informe de visitas de pares académicos durante los procesos de acreditación, Plan de acción y sus responsables, e informes provenientes de la Vicerrectoría de Relaciones y Cooperación Internacional de la Universidad de Cartagena; así como se llevó a cabo una entrevista no estructurada a profesores y grupos de estudiantes pertenecientes al Programa en lo que respecta al subproceso de Movilidad Internacional para identificar sus necesidades y requerimientos con respecto al mismo; y se utilizaron revistas de artículos especializados disponibles en catálogos electrónicos de las bases de datos de la Universidad de Cartagena como Sciencedirect, Scopus, Springer y ESBCOHOST.

\section{Resultados}

\section{Enfoque Sistémico Convergente de la Calidad ESCC}

El ESCC es una herramienta que permite elaborar productos y servicios que cumplen con las necesidades, expectativas y requisitos de los clientes, generando soluciones y evaluando a través de datos y hechos el nivel de evolución y desempeño, el valor agregado, las transformaciones de las operaciones y el nivel de retroalimentación y recursividad del proceso o área intervenido. Los resultados de los doce pasos y su aplicación se resumen en la Figura 1. 
Figura 1. Enfoque Sistémico Convergente de la Calidad aplicado al proceso de Internacionalización del Programa Administración Industrial, Universidad de Cartagena.

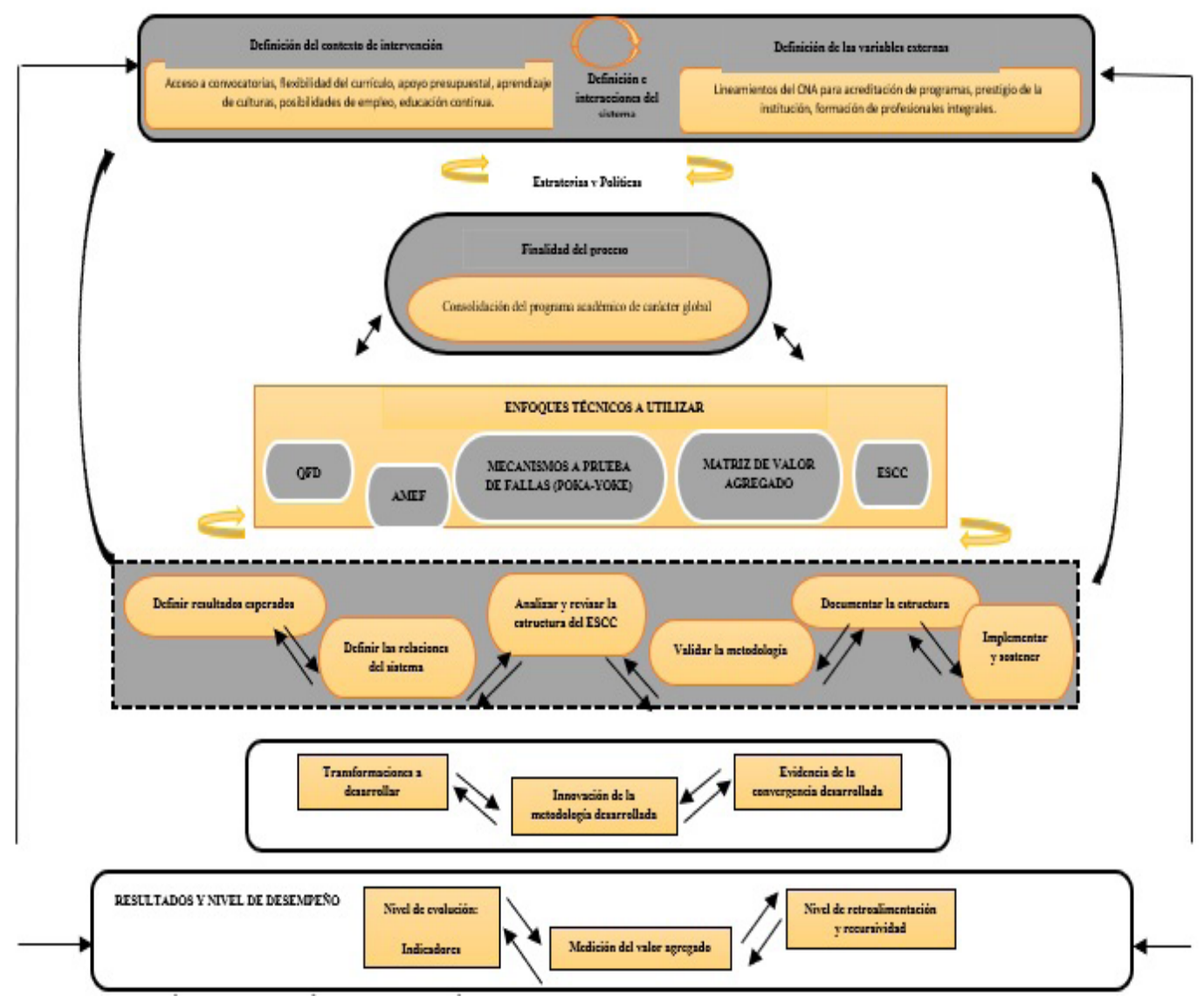

Fuente: Elaboración a partir de Fontalvo [6]

\section{Pasos para operacionalizar la intervención empresarial}

\section{Definición del contexto, de los clientes y sus expectativas}

Para comenzar la aplicación se procedió a modelar el proceso. En la Figura 2 se muestra el diagrama de flujo de la gestión académica de movilidad saliente como ejemplo de las actividades desarrolladas por el programa Administración Industrial dentro del macroproceso Internacionalización, involucrando a los estudiantes, la Vicerrectoría de Relaciones y Cooperación Internacional y el Consejo Académico del Programa en mención. 
Figura 2. Diagrama de flujo. Movilidad Internacio $\neg$ nal
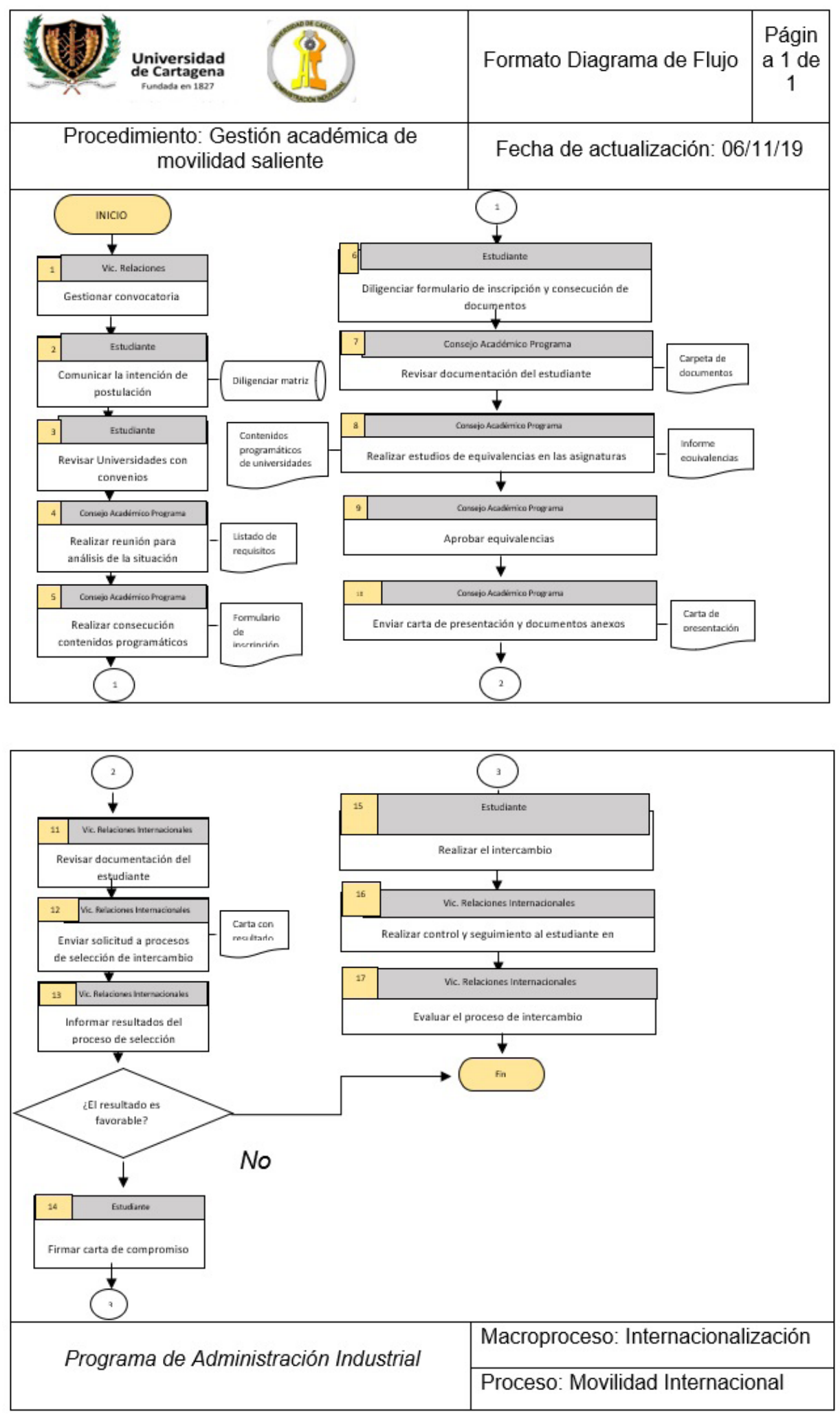

Fuente: Elaboración propia 


\section{Interacción con el contexto}

El subproceso de Movilidad Internacional del Programa Administración Industrial de la Universidad de Cartagena se fundamenta en el objetivo de formar profesionales integrales e insertarlos en la sociedad mundial del conocimiento para lograr alto impacto global y en la comunidad inmediata, a la vez que se incrementa la competitividad en las Instituciones de Educación Superior (IES).

\section{Finalidad del proceso, sistema u organización intervenida.}

La finalidad del proceso (objeto de estudio) de Movilidad Internacional como parte del macroproceso de Internacionalización es el de fomentar la cultura de la internacionalización que impacte en la comunidad académica udeceísta, en la ciudad de Cartagena, en la Región Caribe, incluso en Colombia, a través de un enfoque hacia la multiculturalidad, el bilingüismo y la acreditación Internacional.

\section{Causas de intervención o especificación de necesidades y expectativas del cliente interno o externo}

El Consejo Nacional de Acreditación dentro de sus lineamientos para la acreditación de programas académicos establece que uno de los factores a evaluar corresponde a la visibilidad nacional e internacional, por tanto, la Universidad de Cartagena y sus programas académicos fijaron planes de acción para alcanzar metas alcanzables en cuanto a este factor. En general, los estudiantes manifestaron puntos importantes a tener en cuenta para el desarrollo de un proceso de Movilidad Internacional, como son: fácil acceso a convocatorias, flexibilidad del currículo, apoyo presupuestal, aprendizaje de culturas, posibilidades de empleo en el exterior y las oportunidades de educación continua.

\section{Enfoques técnicos y herramientas de calidad a utilizar}

Las herramientas de calidad a utilizar constituyen un soporte para visualizar el proceso actual y posibles acciones para su optimización a través de perspectivas diversas, como el despliegue de la función calidad (QFD), análisis de modelo, efecto y falla (AMEF); mecanismos a prueba de fallas (poka-yoke) y la matriz de valor agregado.

\section{Despliegue de la función calidad (QFD)}

Con el fin de alcanzar ventajas competitivas se desarrolla el despliegue de la función calidad a través de la herramienta denominada "la casa de la calidad", tal como se muestra en la Figura 3, comparando el programa de Administración Industrial de la Universidad de Cartagena con programas similares, llegando a la conclusión que se puede mejorar el apoyo con el presupuesto a los estudiantes y la flexibilidad del currículo. Dichos programas forman parte de instituciones de Educación Superior según el Ranking de las mejores Universidades de Colombia según los resultados en las pruebas Saber Pro. 
Los pasos a seguir para la elaboración de esta herramienta son:

- Definir los requerimientos de los clientes.

- Definir los requerimientos técnicos.

- Determinar la relación entre los requerimientos del cliente y los técnicos (9 para relaciones fuertes, para relaciones medias y 1 para relaciones débiles)

- Definir la importancia de cada requerimiento de los clientes.

- Evaluar la importancia técnica

- Determinar la dirección de mejora

- Determinar los valores objetivos para los requerimientos técnicos

- Realizar la evaluación técnica

- Realizar la evaluación competitiva

- Definir las interacciones de los requerimientos técnicos

Figura 3. Casa de la calidad. Subproceso Movilidad Internacional Programa Administración Industrial.

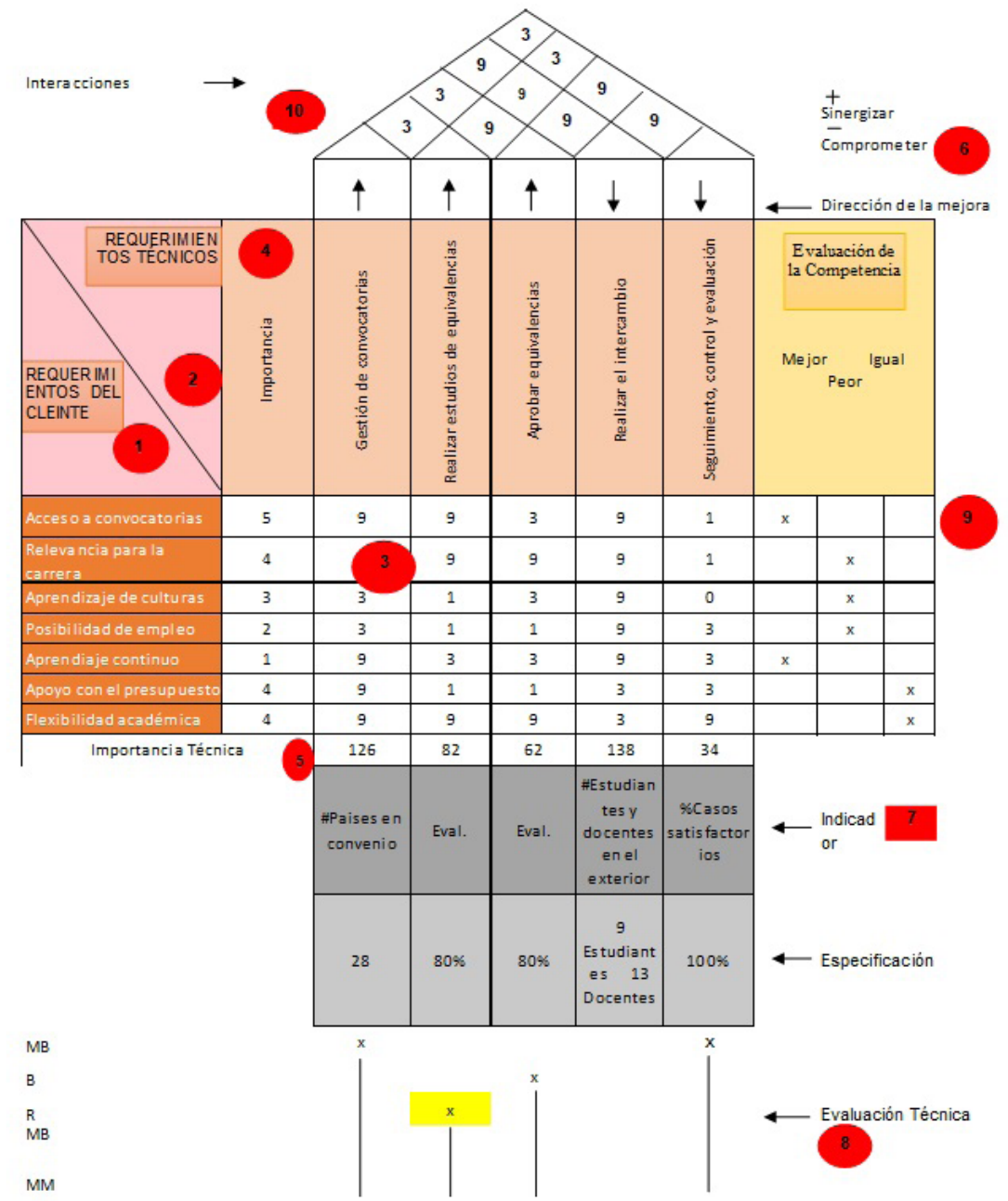

Fuente: Elaboración propia 


\section{Análisis de modelo, efecto y falla (AMEF)}

Siguiendo con el proceso Movilidad Internacional dentro del macroproceso Internacionalización, es importante la elaboración del AMEF, O FMEA para una correcta prevención de los posibles modos de falla, detectándolos a tiempo con el fin de establecer los controles adecuados que eviten la ocurrencia de defectos, dándole prioridad a los pasos del proceso con mayor NPR: aprobar equivalencias y realizar el intercambio, según se muestra en la Figura 4.

Figura 4. Análisis de modo, efecto y falla AMEF.

\begin{tabular}{|c|c|c|c|c|c|c|c|c|c|c|}
\hline $\begin{array}{l}\text { Nombre de } \\
\text { Proceso o } \\
\text { Producto: }\end{array}$ & Internacionalización & & & & & & & & Preparado por: & $\begin{array}{l}\text { González } \\
\text { Fontalvo }\end{array}$ \\
\hline Encargado: & $\begin{array}{l}\text { Programa Adm } \\
\text { Industrial }\end{array}$ & & & & & & & & $\begin{array}{l}\text { FMEA Fecha } \\
\text { (Orig): }\end{array}$ & 43785 \\
\hline $\begin{array}{l}\text { Pasos Clave } \\
\text { del Proceso }\end{array}$ & $\begin{array}{c}\text { Modos de Falla } \\
\text { Potenciales }\end{array}$ & $\begin{array}{c}\text { Efectos } \\
\text { de Fallas } \\
\text { Potenciales }\end{array}$ & SEV & $\begin{array}{c}\text { Causas } \\
\text { Potenciales }\end{array}$ & OCU & $\begin{array}{l}\text { Controles de } \\
\text { Ocurrencia }\end{array}$ & DET & NPR & $\begin{array}{c}\text { Acciones } \\
\text { Recomendadas }\end{array}$ & Resp. \\
\hline $\begin{array}{l}\text { ¿Cuál es el } \\
\text { paso del } \\
\text { proceso? }\end{array}$ & $\begin{array}{l}\text { ¿De qué maneras } \\
\text { puede fallar dicho } \\
\text { paso del proceso? }\end{array}$ & $\begin{array}{l}\text { ¿Cuál es el } \\
\text { impacto de las } \\
\text { variables de } \\
\text { los pasos clave } \\
\text { cuando hay un } \\
\text { fallo (cliente o } \\
\text { requerimientos } \\
\text { internos)? }\end{array}$ & $\begin{array}{l}\text { ¿Qué tan } \\
\text { severo es } \\
\text { el efecto } \\
\text { para el } \\
\text { cliente? }\end{array}$ & $\begin{array}{l}\text { ¿Qué causa } \\
\text { que el paso } \\
\text { clave falle? }\end{array}$ & $\begin{array}{l}\text { ¿Qué tan } \\
\text { seguido } \\
\text { ocurre la } \\
\text { causa o } \\
\text { Modo de } \\
\text { Fallo? }\end{array}$ & $\begin{array}{l}\text { ¿Cuáles son } \\
\text { los controles } \\
\text { existentes y } \\
\text { procedimientos } \\
\text { preventivos de } \\
\text { Causa o Modo } \\
\text { de Falla? }\end{array}$ & $\begin{array}{c}\text { ¿Qué } \\
\text { tan bien } \\
\text { pueden } \\
\text { detectar } \\
\text { la Causa o } \\
\text { Modo de } \\
\text { Falla? }\end{array}$ & $\mathrm{S}^{*} \mathrm{O}^{*} \mathrm{D}$ & $\begin{array}{c}\text { ¿Cuáles son } \\
\text { las acciones } \\
\text { para reducir } \\
\text { la Ocurrencia } \\
\text { de la Causa } \\
\text { o mejorar la } \\
\text { Detección? }\end{array}$ & $\begin{array}{l}\text { ¿Quién es } \\
\text { responsible de } \\
\text { las acciones } \\
\text { recomendadas? }\end{array}$ \\
\hline $\begin{array}{l}\text { Gestión de } \\
\text { convocatorias }\end{array}$ & $\begin{array}{c}\text { Dominio insuficiente } \\
\text { de inglés/francés y/o } \\
\text { alemán }\end{array}$ & $\begin{array}{c}\text { Disminución } \\
\text { de convenios } \\
\text { vigentes }\end{array}$ & 6 & $\begin{array}{c}\text { Poca } \\
\text { capacitación } \\
\text { y } \\
\text { actualizaión } \\
\text { del personal }\end{array}$ & 2 & $\begin{array}{c}\text { Capacitaciones } \\
\qquad y \\
\text { actualizaciones }\end{array}$ & 2 & 24 & $\begin{array}{c}\text { Realizar } \\
\text { capacitaciones } \\
\text { y evaluar lo } \\
\text { aprendido }\end{array}$ & $\begin{array}{c}\text { Secretaría } \\
\text { General }\end{array}$ \\
\hline $\begin{array}{c}\text { Realizar } \\
\text { estudios de } \\
\text { equivalencias }\end{array}$ & $\begin{array}{l}\text { Gestión inoportuna } \\
\text { del comité académico }\end{array}$ & $\begin{array}{l}\text { Posibilidad de } \\
\text { pérdida de una } \\
\text { convocatoria }\end{array}$ & 10 & $\begin{array}{c}\text { Mala gestión } \\
\text { del tiempo } \\
\text { del comité } \\
\text { académico }\end{array}$ & 2 & $\begin{array}{l}\text { Informe de } \\
\text { reuniones } \\
\text { comité }\end{array}$ & 1 & 20 & $\begin{array}{l}\text { Control de } \\
\text { reuniones } \\
\text { comité }\end{array}$ & $\begin{array}{c}\text { Comité } \\
\text { Académico } \\
\text { Programa }\end{array}$ \\
\hline $\begin{array}{c}\text { Aprobar } \\
\text { equivalencias }\end{array}$ & $\begin{array}{c}\text { Materias no } \\
\text { homologables }\end{array}$ & $\begin{array}{c}\text { Reducción del } \\
\text { atractivo hacia } \\
\text { la movilidad }\end{array}$ & 7 & $\begin{array}{c}\text { Baja } \\
\text { flexibilidad } \\
\text { del } \\
\text { currículum }\end{array}$ & 6 & $\begin{array}{l}\text { Reforma } \\
\text { pénsum }\end{array}$ & 4 & 168 & $\begin{array}{l}\text { Rediseñar } \\
\text { el plan de } \\
\text { estudios }\end{array}$ & $\begin{array}{c}\text { Comité } \\
\text { Académico } \\
\text { Programa }\end{array}$ \\
\hline $\begin{array}{l}\text { Realizar el } \\
\text { intercambio }\end{array}$ & $\begin{array}{c}\text { No consecución de } \\
\text { presupuesto }\end{array}$ & $\begin{array}{l}\text { Desmotivación } \\
\text { del estudiante }\end{array}$ & 8 & $\begin{array}{c}\text { Mala } \\
\text { destinación } \\
\text { de recursos } \\
\text { para } \\
\text { movilidad } \\
\text { académica }\end{array}$ & 7 & $\begin{array}{l}\text { Pronóstico de } \\
\text { presupuesto }\end{array}$ & 2 & 112 & $\begin{array}{l}\text { Estudio } \\
\text { de gastos } \\
\text { relacionados } \\
\text { con la } \\
\text { movilidad }\end{array}$ & $\begin{array}{l}\text { Vicerrectoría } \\
\text { de Relaciones } \\
\text { y Cooperación } \\
\text { Internacional }\end{array}$ \\
\hline $\begin{array}{l}\text { Seguimiento, } \\
\text { control y } \\
\text { evaluación }\end{array}$ & $\begin{array}{l}\text { Procedimiento } \\
\text { de seguimiento, } \\
\text { evaluación y control } \\
\text { ineficiente }\end{array}$ & $\begin{array}{l}\text { Pérdida de } \\
\text { información } \\
\text { valiosa para } \\
\text { posteriores } \\
\text { convocatorias }\end{array}$ & 6 & $\begin{array}{l}\text { Omisión de } \\
\text { elementos } \\
\text { por evaluar }\end{array}$ & 1 & $\begin{array}{c}\text { Buena } \\
\text { comunicación } \\
\text { con el } \\
\text { estudiante }\end{array}$ & 2 & 12 & $\begin{array}{c}\text { Mejorar la } \\
\text { comunicación } \\
\text { con los } \\
\text { estudiantes en } \\
\text { intercambio }\end{array}$ & $\begin{array}{c}\text { Vicerrectoría } \\
\text { de Relaciones } \\
\text { y Cooperación } \\
\text { Internacional } \\
\text { y comité } \\
\text { académico } \\
\text { Programa }\end{array}$ \\
\hline
\end{tabular}

\section{Fuente: Elaboración propia}




\section{Mecanismos a prueba de fallas (Poka-Yoke)}

La herramienta de calidad diseñada por Fontalvo [6] denominada "matriz de análisis para mecanismos a prueba de fallas" apoyada en los planteamiento de shingo Shingeo garantiza que, una vez identificada la causa de un error, nunca se vuelva a presentar a través de inspecciones sucesivas, la auto-inspección e inspección en la fuente con el fin de contribuir a la mejora continua de los procesos. Para este caso, como se ve reflejado en la Tabla 2, los mecanismos a prueba de fallas van desde capacitaciones y mejoras en la comunicación con los estudiantes en intercambio hasta el rediseño del plan de estudios con tal de que las asignaturas sean homologables en universidades extranjeras.

Tabla 2. Matriz de análisis para los mecanismos a prueba de fallas (Poka-Yoke)

\begin{tabular}{|c|c|c|c|c|c|c|}
\hline \multirow{2}{*}{\multicolumn{4}{|c|}{ Matriz para los mecanismos a prueba de fallas }} & \multicolumn{3}{|c|}{ Código } \\
\hline & & & & \multicolumn{3}{|c|}{ Página 1 de 1} \\
\hline \multicolumn{7}{|c|}{ Macroproceso: Internacionalización } \\
\hline \multicolumn{7}{|c|}{ Proceso: Movilidad Internacional } \\
\hline Actividad & $\begin{array}{l}\text { Mecanismo a prueba de } \\
\text { falla }\end{array}$ & \multicolumn{3}{|c|}{ Tipo de control } & \multicolumn{2}{|c|}{ Tipo de falla } \\
\hline \multirow{3}{*}{ Gestión de convocatorias } & \multirow[t]{2}{*}{$\begin{array}{l}\text { Realizar capacitaciones y evaluar } \\
\text { lo aprendido }\end{array}$} & Sucesiva & $\begin{array}{l}\text { Auto } \\
\text { inspección }\end{array}$ & Fuente & Servidor & Cliente \\
\hline & & & & $x$ & $x$ & \\
\hline & $\begin{array}{l}\text { Asistir a eventos de entidades } \\
\text { de intercambio o universidades } \\
\text { internacionales }\end{array}$ & $x$ & & & $x$ & \\
\hline \multirow{2}{*}{$\begin{array}{l}\text { Realizar estudios de } \\
\text { equivalencias }\end{array}$} & Control de reuniones comité & $x$ & & & $x$ & \\
\hline & $\begin{array}{l}\text { Monitoreo de programas en el } \\
\text { exterior }\end{array}$ & $x$ & & & $\mathrm{x}$ & \\
\hline Aprobar equivalencias & Rediseñar el plan de estudios & & $x$ & & $x$ & \\
\hline \multirow{2}{*}{ Realizar el intercambio } & $\begin{array}{l}\text { Estudio de gastos relacionados } \\
\text { con la movilidad }\end{array}$ & & & $\mathrm{x}$ & $\mathrm{x}$ & $x$ \\
\hline & $\begin{array}{l}\text { Charlas informativas antes del } \\
\text { intercambio }\end{array}$ & & & $x$ & $x$ & $\mathrm{x}$ \\
\hline \multirow{2}{*}{$\begin{array}{l}\text { Seguimiento, control y } \\
\text { evaluación }\end{array}$} & $\begin{array}{l}\text { Mejorar la comunicación con los } \\
\text { estudiantes en intercambio }\end{array}$ & $x$ & & & $\mathrm{x}$ & \\
\hline & $\begin{array}{l}\text { Realizar las mejoras necesarias } \\
\text { en los procedimientos de } \\
\text { seguimiento }\end{array}$ & & $x$ & & $x$ & \\
\hline
\end{tabular}

Fuente: Elaboración a partir de Fontalvo [6]

\section{Matriz de valor agregado}

La matriz de valor agregado constituye una herramienta para la valoración de la aplicación del enfoque sistémico de la calidad o cualquier enfoque técnico o herramienta de calidad utilizado en un proceso o área específica. Se tienen en cuenta las operaciones del proceso más relevantes para la organización. Es decir, las que agregan valor a los clientes y a la organización misma, así como las dimensiones de calidad y actividades de los productos o servicios que satisfagan los requerimientos del cliente, como lo muestra la Tabla 3, donde se ve reflejado el valor agregado de las actividades facilitadoras de la Movilidad Académica Internacional a las partes interesadas del proceso. 
Tabla 3. Matriz de valor agregado. Proceso Movilidad Internacional

\begin{tabular}{|c|c|c|c|c|}
\hline \multirow{2}{*}{\multicolumn{4}{|c|}{ Matriz de valor agregado }} & Código \\
\hline & & & & Página 1 de 1 \\
\hline \multirow{2}{*}{$\begin{array}{c}\text { Actividad del proceso } \\
\text { intervenido }\end{array}$} & \multirow{2}{*}{$\begin{array}{c}\text { Valor agregado por } \\
\text { actividad }\end{array}$} & \multicolumn{3}{|c|}{ Tipo de valor generado } \\
\hline & & Valor económico & Valor social & Valor tecnológico \\
\hline Gestión de convocatorias & $\begin{array}{c}\text { Acceso a convocatorias } \\
\text { internacionales de redes de } \\
\text { investigación, intercambios y } \\
\text { promoción del conocimiento a } \\
\text { nivel internacional }\end{array}$ & $\begin{array}{c}\text { Obtención de } \\
\text { convocatorias para becas }\end{array}$ & $\begin{array}{l}\text { Intercambio } \\
\text { de culturas, } \\
\text { experiencias y } \\
\text { conocimiento } \\
\text { académico }\end{array}$ & $\begin{array}{l}\text { Construcción de } \\
\text { conocimiento en redes de } \\
\text { investigación internacional }\end{array}$ \\
\hline $\begin{array}{l}\text { Realizar estudios de } \\
\text { equivalencias }\end{array}$ & $\begin{array}{l}\text { Homologación de estudios en } \\
\text { el exterior }\end{array}$ & & $\begin{array}{c}\text { Equivalencias } \\
\text { en los planes de } \\
\text { estudio }\end{array}$ & $\begin{array}{l}\text { Aprendizaje de tendencias } \\
\text { internacionales en el } \\
\text { campo de estudio }\end{array}$ \\
\hline Aprobar equivalencias & Flexibilidad del currículo & & $\begin{array}{l}\text { Mayor impacto y } \\
\text { motivación en la } \\
\text { comunidad }\end{array}$ & $\begin{array}{l}\text { Eficiencia de los procesos } \\
\text { de movilidad académica }\end{array}$ \\
\hline Realizar el intercambio & $\begin{array}{l}\text { Formación integral de los } \\
\text { profesionales como ciudadanos } \\
\text { del mundo }\end{array}$ & $\begin{array}{l}\text { Obtención de recursos } \\
\text { para intercambios } \\
\text { académicos }\end{array}$ & $\begin{array}{l}\text { Intercambio } \\
\text { cultural }\end{array}$ & $\begin{array}{c}\text { Conocimientos adaptados } \\
\text { a otras realidades }\end{array}$ \\
\hline $\begin{array}{l}\text { Seguimiento, control y } \\
\text { evaluación }\end{array}$ & $\begin{array}{l}\text { Acceso a la experiencia del } \\
\text { estudiante en el exterior, } \\
\text { monitoreo de nuevos } \\
\text { conocimientos adquiridos }\end{array}$ & & $\begin{array}{l}\text { Medición de la } \\
\text { experiencia del } \\
\text { intercambio }\end{array}$ & $\begin{array}{c}\text { Adquisición de } \\
\text { competencias de carácter } \\
\text { intercultural }\end{array}$ \\
\hline
\end{tabular}

Fuente: Elaboración propia de los autores basado en Enfoque Sistémico Convergente De La Calidad: Una Visión Práctica [18]

\section{Metodología propuesta de generación de valor}

A través de ESCC se busca la implementación de un modelo que articule distintas herramientas y técnicas de calidad con el fin de mitigar fallas en los procesos actuales, generar valor agregado en el Programa de Administración Industrial, y satisfacer los requerimientos, necesidades y expectativas de los clientes; que en este caso son los estudiantes. En primer lugar, se sintetiza en un diagrama de flujo de las operaciones o actividades que se realizan para ejecutar el subproceso de movilidad internacional, luego se evalúa el cumplimiento de las necesidades de los clientes a través de las capacidades técnicas y se compara con la competencia en el análisis de la herramienta QFD. Por otro lado, se priorizan actividades con mayor probabilidad de falla y se generan acciones de control para evitar dichas fallas en el AMEF; se analizan las fallas y los orígenes de las mismas para controlar y realizar un seguimiento adecuado a los responsables en el sistema POKA-YOKE, así como se establecen y caracterizan las actividades del proceso y el valor agregado que generan para la comunidad y la organización. 


\section{Transformaciones a desarrollar}

Por medio de indicadores como el número de convenios realizados con universidades y entidades de cooperación internacional, número de estudiantes y docentes en el exterior, porcentaje de casos exitosos de movilidad y de materias aprobadas u homologadas para hacer efectivo el intercambio con universidades extranjeras, se mide el desempeño y se monitorea el valor agregado de las actividades que componen el proceso de movilidad internacional; toda vez que contribuye al mejoramiento de la calidad y competitividad, se mantienen satisfechos a los clientes y se motiva a la comunidad académica a hacer parte del proceso, con un enfoque de calidad sistémica y mejoramiento continuo fortaleciendo la flexibilidad del currículo, el apoyo en la consecución de presupuesto para movilidad académica y soporte a través de la formación en mínimo una segunda lengua.

\section{Innovación de la metodología desarrollada}

El proceso de innovación en la metodología está asociado con la articulación sistémica de las herramientas utilizadas como son los procesos, el QFD, el AMEF, los mecanismos a prueba de Fallas. Lo cual se propone de manera articulada para planificar, controlar y mejorar e impactar en la sociedad. Generando un servicio de internacionalización innovador del programa que impacte y tenga aceptación en la comunidad académica y a su vez contribuya con la acreditación del programa.

\section{Convergencia del sistema}

Tomando como base las necesidades de los clientes y del entorno externo se deben planificar las actividades de los procesos, estableciendo recursos y metas alcanzables y verificables, así como responsables de su consecución; dando pleno cumplimiento a la normatividad legal vigente y a las políticas establecidas por la Universidad de Cartagena. Apoyado con las herramientas propuestas en esta investigación.

\section{Nivel de desempeño y evolución del área de intervención}

La evaluación del desempeño del proceso, a través de los indicadores antes mencionados, se elaboró continuamente, con el objetivo de detectar falencias en alguna de las actividades y mitigar los efectos que se pudiesen generar de acuerdo a las especificaciones y requerimientos de clientes actuales y/o potenciales.

\section{Valor agregado del proceso intervenido}

La movilidad internacional como parte del proceso de internacionalización constituye un elemento de alto impacto social, tecnológico y en parte económico para sus partes interesadas. Determinando la importancia para la asignación de recursos al programa. Así atrayendo recursos de organizaciones internacionales enfocadas en la cooperación e investigación conjunta de carácter internacional. Todo esto, en última instancia, es materia prima para generación de conocimiento, que sin la gestión realizada en este proceso muy difícilmente sería visible a los estudiantes y docentes con potencial para el desarrollo científico. 


\section{Nivel de retroalimentación y recursividad}

Con el fin de garantizar los procesos de mejora en el proceso de internacionalización, es fundamental considerar los indicadores de desempeño y evolución propuestos, Así como las percepciones de todas las partes interesadas asociadas con el proceso de internacionalización de tal manera con con la retroalimentación y recursividad captada del entorno, se puedan redireccionar las actividades débiles y aprovechar los buenos desempeños asociados con los indicadores propuestos en esta investigación. De tal forma que se puedan satisfacer las necesidades y expectativas de los usuarios que hacen parte del proceso de internacionalización del programa de Administración Industrial.

\section{Discusión}

En la actualidad no existen estudios que hagan referencia al proceso de internacionalización en instituciones de educación superior y su optimización bajo enfoques de calidad sistémica. De modo general Minocha, Shiel, \& Histrov [19] realizan una aproximación al enfoque de este artículo al sugerir que las instituciones académicas deben considerar si el currículo y los procesos son limitados y limitantes a favor de una lente cultural estrecha; Popescu [20] en otro estudio concluye que la implementación del modelo de enfoque estratégico de calidad puede conducir a: a) Permitir opciones de aprendizaje más variadas para los estudiantes y adaptarse más fuerte a sus necesidades; b) Permitir un mayor nivel de flexibilidad a las universidades a los cambios que ocurren a nivel social; c) Brindar oportunidades para la movilidad social, cubrir adecuadamente las necesidades en diferentes mercados laborales (por ejemplo, demanda de diferentes especialidades) y brindar las oportunidades necesarias para la innovación donde subyace la relación no dicotómica entre creatividad, estandarización y diversidad. Métodos como el enfoque sistémico, análisis, síntesis, métodos especiales de investigación, método de evaluaciones de expertos, observación, análisis de prácticas educativas, encuesta del personal pedagógico y directivo de la organización educativa, y métodos estadísticos son plateados en [21] para darle importancia al papel del desarrollo del personal directivo en la estructura del sistema de gestión de calidad de las organizaciones educativas; se fundamentan las oportunidades, objetivos y tareas de desarrollo del personal gerencial como un subsistema del sistema de gestión de calidad de las organizaciones educativas y aporta algunas recomendaciones para construir el sistema de desarrollo del personal directivo en el contexto de los principios del sistema de gestión de calidad de las organizaciones educativas. Por otro lado en [22, 23], plantean que la resolución de problemas en sistemas complejos requiere comprender los tipos de relaciones entre los actores del sistema, así como sus ciclos de influencia e interacción, para identificar formas de acción en puntos que pueden promover interacciones en el sentido deseado.

Además, según Thomas [24] adoptar una perspectiva de sistemas completos podría generar información sobre una amplia gama de puntos de inflexión del sistema o umbrales críticos que conllevan a cambios cualitativamente diferentes, discontinuos y potencialmente irreversibles, por tanto, es necesario comprender las características de estos puntos para crear, evitar o gestionar sus consecuencias sistémicas, para bien o para mal. 
Por otro lado, el pensamiento sistémico, permite comprender realmente cómo son los procesos de trabajo, la toma de decisiones y el aprendizaje que puede ocurrir dentro del sistema organizacional, razón por la que su aplicabilidad en los procesos de internacionalización es fundamental para planificar, controlar y mejorar estos procesos en el programa objeto de estudio y de esta manera llenar las necesidades y expectativas de las partes interesadas. De igual manera otras investigaciones [25, 26, 27, 28, 29] desde perspectivas sistémicas a mostrado la pertinencia para establecer métricas, que permitan evaluar el desempeño y evolución de los procesos como en esta investigación.

\section{Conclusiones}

Es posible modelar los procesos y los servicios inmersos en el macroproceso de internacionalización del programa de Administración Industrial de la Universidad de Cartagena con el fin de garantizar la satisfacción plena de las necesidades de sus clientes, a través de un Enfoque Sistémico Convergente de la Calidad (ESCC), incluyendo herramientas de calidad tales como el Despliegue de la Función Calidad (QFD), el Análisis de Modo, Efecto y Falla (AMEF); los Mecanismos a Prueba de Fallas (Poka-Yoke) y la Matriz de Valor Agregado, tomando como objeto de estudio el proceso de movilidad internacional, las conclusiones de esta investigación fueron:

- El programa debe priorizar en el proceso de internacionalización, en la participación en convocatorias, el grado de relevancia y aprendizaje que representa la experiencia de la movilidad, el aprendizaje de otra cultura, el apoyo institucional con los gastos presupuestados, la flexibilidad del currículo, así como la posibilidad de empleo y educación continua en el exterior.

- Se debe priorizar la aprobación de equivalencias y la realización de intercambios como puntos clave del proceso de movilidad internacional para una correcta prevención de los posibles modos de falla, detectándolos a tiempo con el fin de establecer los controles adecuados que eviten la ocurrencia de fallas en el proceso.

- Se deben realizar capacitaciones para el aprendizaje y fortalecimiento de, mínimo, una segunda lengua, evaluando lo aprendido en las mismas; asistir a eventos de entidades de intercambio o universidades internacionales, llevar un control de las reuniones del comité académico del Programa, monitorear de forma constante los planes de estudios ofrecidos por instituciones extranjeras para facilitar los estudios de equivalencias; realizar estudios relacionados con los gastos requeridos para llevar a cabo la movilidad internacional, charlas informativas antes de los intercambios, ajustar los procedimientos de seguimiento a la vez que se deben establecer y mantener mejores canales de comunicación con los estudiantes que accedan a cualquier convocatoria de movilidad. Todo esto con el objetivo de prevenir anticipadamente posibles fallas que afecten perjudicialmente la efectividad del proceso.

- Por último, el proceso de movilidad internacional visto desde un Enfoque Sistémico genera valor agregado para el Programa de Administración Industrial, en tanto brinda, desde cada una de sus actividades y su mejoramiento continuo, estructuras de planificación y control. Garantizando el intercambio de conocimientos a nivel internacional y a la formación de profesionales integrales ciudadanos del mundo. 


\section{Referencias bibliográficas}

1. CNA, “Lineamientos para la acreditación de programas de pregrado", 2013.

2. Vicerrectoría de Relaciones y Cooperación Internacional, “Plan Institucional de Internacionalización”, 2018.

3. Programa Administración Industrial, “Plan de Desarrollo Administración Industrial 2014-2018”, 2014.

4. A. Abrahamsen, P. M. Allen, \& X. Barandiaran, "Philosophy of Complex Systems", Cliff Hooker ed., vol. 10, 2011. DOI: https://doi.org/10.1016/B978-0-444-52076-0.50028-67

5. D. Eriksson, "A Principal Exposition of Jean-Louis Le Moigne's Systemic Theory", Cybernetics \& Human Knowing, vol. 4, no. 2, 1997

6. T. Fontalvo. "Propuesta de intervención empresarial para la generación de valor en las organizaciones", Revista ADMpg Gestão Estratégica, Ponta Grossa, vol. 1, no. 1, pp.161-166, 2008.

7. L. Berry, V. A. Zeithaml, \& A. Parasuraman, "Quality counts in services, too", Business Horizons, vol. 28, no. 3, pp. 44-52, 1985. DOI: https://doi.org/10.1016/0007-6813(85)90008-4

8. B. M. Almohaimmeed, "Internal service quality and external service quality using two versions of servqual scale: An empirical evidence from five malls in the capital city of Saudi Arabia", Business: Theory and Practice, vol. 20, pp. 158-169. doi:10.3846/BTP.2019.15

9. L. Businska, \& M. Kirikova, The Goal-Based Selection of the Business Process Modeling Language, pp. 307-316, 2016. doi:https://doi.org/10.1007/978-3-319-48393-1_22

10. A. Cherchata, I. Popovychenko, U. Andrusiv, L. Simkiv, O. Kliukha, \& O. Horai, "A methodology for analysis and assessment of business processes of Ukrainian enterprises". Management Science Letters, vol. 10, no. 3, pp. 631-640. 2019. doi:10.5267/j.msl.2019.9.016

11. C. Y. Chan, K.-S. Chin, C. Chan, \& K. L. Tsui, "An analysis of passengers' ride needs of urban rail transit services: application of quality function deployment". International Journal of Quality Innovation, 2019. DOI: https://doi.org/10.1186/s40887-019-0033-9

12. Y. Akao, \& G. Mazur, "Using QFD to assure QS-9000 compliance", 1998.

13. E. Vezzetti, F. Marcolin, \& A. L. Guerra, "QFD 3D: a new C-shaped matrix diagram quality approach", International Journal of Quality and Reliability Management, vol. 33, no. 2, pp. 178-196, 2016. doi:10.1108/ IJQRM-07-2013-0112

14. Z. Xu, Y. D. Munro, \& Y. Wang, "A data-driven approach for constructing the component-failure mode matrix for FMEA", Journal of Intelligent Manufacturing, pp. 1-17, 2019. DOI: https://doi.org/10.1007/ s10845-019-01466-z

15. H.C. Liu, Y.-Z. Chen, J.-X. You, \& H. Li, "Risk evaluation in failure mode and effects analysis using fuzzy digraph and matrix approach". Journal of Intelligent Manufacturing, vol. 27, no 4, pp. 805-816, 2016. DOI: https://doi.org/10.1007/s10845-014-0915-6

16. M. Vinod, S. R. Devadasan, D. T. Sunil, \& V. M. Thilak, "Six Sigma through Poka-Yoke: a navigation through literature arena", The International Journal of Advanced Manufacturing Technology, vol. 81, pp. 315-327, 2015. DOI: https://doi.org/10.1007/s00170-015-7217-9

17. S. Shingo, E-book: "The Sayings of Shigeo Shingo: Key Strategies for Plant Improvement", 2017. DOI: https://doi.org/10.1201/9780203736043

18. T. Fontalvo, J. Vergara, \& J. Morelos, "Enfoque Sistémico Convergente De La Calidad: Una Visión Práctica", Corporación Para La Gestión Del Conocimiento Asesores Del 2000 vol. 500, 2011. 
19. S. Minocha, C., Shiel, \& D. Histrov, "International academic staff in UK higher education: campus internationalisation and innovation in academic practice", Journal of Further and Higher Education, vol. 43, no. 7, pp. 942-958, 2019. doi:https://doi-org.unicartagena.basesdedatosezproxy.com/10.1080/ 0309877X.2018.1429582

20. L. G. Popescu, "From standardization to diversification in Romanian higher education institutions by quality strategic approach". Vision 2020: Innovation, Development Sustainability, and Economic Growth - Proceedings of the 21st International Business Information Management Association Conference, vol. 2, pp. 1046-1056, 2013.

21. V. Tarasenko, "Peculiarities of Development of Managerial Personnel in the Conditions of the System of Quality Management of an Educational Organization". E. G. Popkova, "Growth Poles of the Global Economy: Emergence", Changes and Future Perspectives pp. 1149-1158, 2019. doi:https://doi. org/10.1007/978-3-030-15160-7_116

22. G. Vaccaro, \& A. Scavarda, "Quality of the public health system: A systemic comprehension in Brazilian southern region", Gestão \& Produção, vol. 26, no.. 1, 2019. DOI: http://dx.doi.org/10.1590/0104$530 \times 1626-19$

23. A. Hussein, \& K. Cheng, "An investigation on the engineering process oriented approach to aerospace quality assurance compliance in manufacturing SMEs". 15th International Conference on Manufacturing Research, ICMR, pp. 452-457, 2017.

24. $\quad$ N. J. Thomas, "Sustainability marketing. The need for a realistic whole systems approach". Journal of Marketing Management, vol. 34, pp.1530-1556, 2018. DOI: https://doi.org.unicartagena. basesdedatosezproxy.com/10.1080/0267257X.2018.1547782

25. T. Fontalvo, "Aplicación de análisis discriminante para evaluar la productividad como resultado de la certificación BASC en las empresas de la ciudad de Cartagena".Contaduría y admnistración, vol. 59, pp.43-62, 2014.

26. T. Fontalvo, J. Vergara, \& F. Maza, “La planeación por escenarios: Revisión de conceptos y propuestas metodológicas".Prosperctiva, vol. 8, pp.21-29, 2010.

27. R. Avellaneda, S. Cabrera, P. A. Martínez, y C. G. Donoso Albarracín, "Apoyo tecnológico para la fidelización y captación de nuevos clientes por medio de una aplicación móvil", Investigación e Innovación en Ingenierías, vol. 5, n.o 1, pp. 92-101, 2017. DOI: https://doi.org/10.17081/invinno.5.1.2618

28. A. L. Contreras Giraldo, "Gestión de la motivación en escenarios organizacionales", Investigación e Innovación en Ingenierías, vol. 6, n.o 1, pp. 84 - 92, 2017. DOI: https://doi.org/10.17081/invinno.6.1.2777

29. T. Fontalvo, "La gestión avanzada de la calidad: metodologías eficaces para el diseño, implementación y mejoramiento de un sistema de gestión de calidad". Corporación para la gestión del conocimiento ASD, 2006. 